\title{
Non-Invasive Rhinosinusitis: Clinical and Microbiological Perspective
}

\author{
Diljot Sandhu ${ }^{1}$, Veenu Gupta ${ }^{1}$, Deepinder Chhina ${ }^{1}$, Manish Munjal ${ }^{2}$ \\ ${ }^{1}$ Department of Microbiology, Dayanand Medical College \& Hospital, Ludhiana, Punjab, India \\ ${ }^{2}$ Department of ENT, Dayanand Medical College \& Hospital, Ludhiana, Punjab, India
}

\section{ABSTRACT}

Objectives: Objectives: Fungal rhinosinusitis is one of the important healthcare problems and its incidence and prevalence are increasing over the past three decades. It affects approximately $20 \%$ of the population at some time in their lives. We aimed to identify and characterize the microorganisms causing non-invasive rhinosinusitis and to correlate the findings with the clinical profile of patients.

Methods: Clinically suspected cases of rhinosinusitis were enrolled in the study and detailed clinical history was taken. Samples like nasal mucosa, nasal crusts, scrapings / excised nasal polyps, and biopsy were collected. The specimens were processed for fungal culture. Isolates were identified as per standard protocols.

Results: A total of 74 patients clinically suspected of rhinosinusitis were enrolled in our study out of which $60.8 \%$ were males. The most common presenting complaint was nasal obstruction $(91.8 \%)$ followed by nasal discharge (77\%). Clinically characterizing, the most common type of non-invasive rhinosinusitis seen in our study was allergic rhinosinusitis $(44.6 \%)$. $\mathrm{KOH}$ positivity was $45.6 \%$ and fungal culture positivity was $30.4 \%$. The most common fungal isolate was Aspergillus flavus.

Conclusion: Continuous and periodic evaluation of the microbiological patterns of isolates is necessary to decrease the potential risk of complications by early institution of appropriate treatment. J Microbiol Infect Dis 2021; 11(1):21 26.

Keywords: Fungal rhinosinusitis, Allergic rhinosinusitis, Aspergillus

\section{INTRODUCTION}

Rhinosinusitis is a group of disorders characterized by inflammation of the mucosa of the nose and the paranasal sinuses. It can be initiated by an inflammatory insult i.e; viral infection of the upper respiratory tract or allergic rhinitis preceded by a bacterial or fungal superinfection [1].

Fungal rhinosinusitis is one of the important healthcare problems and its incidence and prevalence have increased over the past three decades. It affects approximately $20 \%$ of the population at some time of their lives. It occurs in both acute and chronic forms. Chronic rhinosinusitis accounts for more than $90 \%$ of all cases of rhinosinusitis. Out of this, $6-12 \%$ of patients will have fungal growth in the culture or in the histopathological study $[2,3]$.

Prior sinus surgery seems to be a more important risk factor for the development of sinus fungal balls. It has been speculated that sinus fungal ball may develop in any poorly ventilated sinus and that fungal exposure and poor sinus ventilation may be the only risk factors that are required [4].

It is important to identify the causative agent responsible for oto-rhinological diseases in favor of effective treatment. The pattern of organisms varies from place to place and depends upon age, habits of the inhabitants, their immune status, and any co-morbidities. These diseases are now being recognized all over the world because of increased awareness.

Microorganisms are more often recognized as secondary invaders. Bacteria, fungi, or viruses may be involved in some cases but there may be cases with no identifiable pathogenic organisms. North India has been identified as an endemic zone of paranasal mycoses. The most important etiological agents of fungal rhinosinusitis are Aspergillus spp., Alternaria, Candida, Bipolaris, and Curvularia spp. [5].

There is also a strong suspicion of the role of allergy. Antigen-antibody reactions result in the 
release of histamine and other mediators of inflammation, which cause changes in vascular permeability, destabilization of lysosomal membranes causing inflammation, mucosal swelling, and ostia obstruction [6].

FRS causes significant physical symptoms, severe quality of life impairment, and can substantially impair daily functioning. As the incidence of chronic rhinosinusitis has increased over the last decade, the economic effect is expected to be more. Knowledge of the prevailing flora and their susceptibility to antimicrobials will guide the clinician to prescribe an empirical regimen so that more specific management can be provided $[7,8]$. This study has been planned to focus on the microbial profile of these non-invasive rhinological infections and correlate with the etiological diagnosis.

\section{METHODS}

This prospective study was conducted in the Department of Microbiology, Dayanand Medical College and Hospital, Ludhiana, India. Seventyfour clinically suspected cases of rhinological infections of any age and either gender were included. Patients detailed demographic, clinical, and investigation details were collected. The study was approved by Institutional Ethical Committee.

Sample collection and transportation: In cases suspected of rhinosinusitis, specimens like nasal secretions, nasal mucosa, crusts, nasal scrapings, excised nasal polyp/tissue biopsy were collected and transported in saline in a sterile, screw-capped container. The specimens received in the Department of Microbiology were processed as per standard protocols.

For direct examination, $\mathrm{KOH}$ MOUNT preparation was made by taking a drop of 10 $40 \% \mathrm{KOH}$ on the center of the clean slide. The specimen was placed in the $\mathrm{KOH}$ drop and teased with the help of inoculating needles. A cover-slip was placed over the material and the slide was then examined under high power magnification [9].

Specimens were also inoculated on four tubes of Sabouraud dextrose agar (SDA) with and without cycloheximide and incubated at $25{ }^{\circ} \mathrm{C}$ and $37{ }^{\circ} \mathrm{C}$. The SDA slants were examined for growth daily for the first week and twice a week for a subsequent period of 4 weeks.

Fungal growth obtained was identified on the basis of colony morphology, rate of growth, color, texture, pigmentation, and findings on lactophenol cotton blue mount examination.

Cases of rhinosinusitis were characterized into allergic fungal rhinosinusitis (AFRS), acute invasive fungal rhinosinusitis, chronic invasive fungal rhinosinusitis, granulomatous invasive fungal rhinosinusitis, and fungal ball on the basis of clinical presentation, duration of the disease, immunological, histopathological, radiological, and microbiological findings [10].

Data obtained from the study were analyzed by descriptive statistical analysis methods, Frequency distribution, Chi-square/Fischer exact test, Significant $p$-value $<0.05$.

\section{RESULTS}

Out of 74 FRS cases, the most affected age group was 41 - 50 years with 24 (32.4\%) cases while the least affected age group was $0-10$ years with $1(1.4 \%)$ cases.

In our study group, $45(60.8 \%)$ of the population that presented with symptoms of rhinosinusitis were males and $29(39.2 \%)$ were females, with a male: female ratio of $1.5: 1$

The most common presenting complaint was nasal obstruction $68(91.8 \%)$ followed by nasal discharge 57 (77\%), headache $31(41.8 \%)$, and postnasal dip 29 (39.1\%). Features like facial pain and swelling and eye symptoms were seen in fewer cases. History of known allergies was seen in $17(23 \%)$ of patients. The majority of patients were allergic to dust and hay.

Unilateral nasal involvement was seen in the majority of the cases 41 (55.4\%) [Right-sided symptoms in 21 (28.3\%) and left-sided involvement in 20 (27\%) cases followed by bilateral involvement in 33 (44.5\%)].

Among the clinical findings, $31(41.8 \%)$ had deviated nasal septum and 26 (35.1\%) had inferior turbinate hypertrophy. Nasal polyps were seen in $25(33.8 \%)$ of cases.

The most common paranasal sinus involved in rhinosinusitis was the maxillary sinus 58 (78.3\%). Overall $\mathrm{KOH}$ positivity was $21(45.6 \%)$ and fungal culture positivity was $14(30.4 \%)$. The 
correlation was significant between direct examination and culture (Table 1).

The most common isolate among different types of non-invasive rhinosinusitis was Aspergillus flavus 11 (23.9\%) (Table 2).

The most common type of rhinosinusitis seen in our study was allergic RS 33 (44.6\%) Urban population was more affected in all types of rhinosinusitis.

Most of the cases presented with a duration of disease >4 weeks $50(67.5 \%)$ and $72(97.2 \%)$ were immunocompetent. The absolute eosinophil count was raised in 32 (43.2\%) of cases. The most common histopathological finding was inflammatory polyp with allergic mucin $(44.5 \%)$. The most common radiological finding observed was the opacity of sinuses. In $11(14.9 \%)$ of cases no abnormality was observed (Table 3 and 4).

In our study, the most common complication in patients with rhinosinusitis was orbital cellulitis 7 $(9.5 \%)$, followed by mucocele in $3(4 \%)$, septal abscess in $3(4 \%)$ and third cranial nerve palsy in $2(2.7 \%)$ cases.

Table 1. Co-relation of direct examination with culture in non-invasive rhinosinusitis cases $(n=36)$.

\begin{tabular}{lcc}
\hline $\begin{array}{l}\text { Direct examination } \\
\text { (KOH results) }\end{array}$ & \multicolumn{2}{c}{$\begin{array}{c}\text { Culture positive (growth on } \\
\text { SDA }\end{array}$} \\
& $\begin{array}{l}\text { Positive, } \\
\mathrm{n}=14(\%)\end{array}$ & $\begin{array}{l}\text { Negative, } \\
\mathrm{n}=22(\%)\end{array}$ \\
\hline Positive, $\mathrm{n}=21$ & $14(66.7)$ & $7(33.3)$ \\
Negative, $\mathrm{n}=15$ & $0(0)$ & $15(100.0)$ \\
\hline
\end{tabular}

p value $<0.05$

Out of 74 patients with rhinosinusitis, 13 were managed conservatively with oral antifungal medication, while 61 cases underwent surgery for the management of symptoms and complications.

Table 2: Distribution of fungal isolates among different types of rhinosinusitis $(n=46)$

\begin{tabular}{|c|c|c|c|c|}
\hline Fungal isolates & Allergic RS ( $n=33)$ & Eosinophilic RS ( $n=8)$ & Fungal ball $(n=5)$ & Total $(n=46)$ \\
\hline A. flavus & 5 & 4 & 2 & $11(23.9 \%)$ \\
\hline A. fumigatus & - & 1 & 1 & $2(4.3 \%)$ \\
\hline Mucor spp. & 1 & - & - & $1(2.1 \%)$ \\
\hline Total & $6(18.1 \%)$ & $5(62.5 \%)$ & $3(60 \%)$ & $14(30.4 \%)$ \\
\hline
\end{tabular}

Table 3. Clinical characterization of non-invasive type of rhinosinusitis on the basis of duration of disease, immunological, histopathological, radiological findings.

\begin{tabular}{|c|c|c|c|c|c|c|c|c|c|}
\hline \multirow{2}{*}{$\begin{array}{l}\text { Types of Rhinosinusitis } \\
\text { Time }\end{array}$} & \multicolumn{2}{|c|}{$\begin{array}{l}\text { Duration of } \\
\text { the disease }\end{array}$} & \multicolumn{2}{|c|}{ Immunology } & \multicolumn{2}{|c|}{$\begin{array}{l}\text { Histopathological } \\
\text { findings }\end{array}$} & \multicolumn{2}{|c|}{$\begin{array}{l}\text { Radiological } \\
\text { findings }\end{array}$} & \multirow{2}{*}{$\begin{array}{l}\text { Total } \\
(n=46)\end{array}$} \\
\hline & $\begin{array}{l}<4 \\
\text { wk }\end{array}$ & $\begin{array}{l}>4 \\
\text { wk }\end{array}$ & Yes & No & Yes & No & Yes & No & \\
\hline Allergic RS & 13 & 20 & 23 & 10 & 29 & 4 & 23 & 10 & $n=33$ \\
\hline Eosinophilic RS & 2 & 6 & 8 & - & 4 & 4 & 4 & 4 & $\mathrm{n}=8$ \\
\hline Fungal ball & 1 & 4 & - & 5 & 5 & - & 5 & - & $\mathrm{n}=5$ \\
\hline
\end{tabular}


Table 4. Distribution of non-invasive rhinosinusitis cases on the basis of gender, age, clinical presentation and comorbidities.

\begin{tabular}{|c|c|c|c|}
\hline Clinical presentation/Features & $\begin{array}{c}\text { Allergic } R S n=33 \text {, } \\
(\%)\end{array}$ & $\begin{array}{c}\text { Eosinophilic RS, } \mathrm{n}=8 \\
(\%)\end{array}$ & $\begin{array}{c}\text { Fungal ball, } \mathrm{n}=5 \\
(\%)\end{array}$ \\
\hline Gender (M) & $21(63.6)$ & $5(62.5)$ & $3(60)$ \\
\hline Area wise distribution (Urban) & $21(63.6)$ & $5(62.5)$ & $3(60)$ \\
\hline $11-20$ years & $2(6)$ & $1(12.5)$ & $0(0)$ \\
\hline $21-30$ years & $6(18.1)$ & $3(37.5)$ & $1(20)$ \\
\hline $31-40$ years & $3(9)$ & $2(25.0)$ & $0(0)$ \\
\hline \multicolumn{4}{|l|}{ Age } \\
\hline $41-50$ years & $14(42.4)$ & $1(12.5)$ & $3(60)$ \\
\hline $51-60$ years & $6(18.1)$ & $0(0)$ & $1(20)$ \\
\hline $60-70$ years & $2(6)$ & $1(12.5)$ & $0(0)$ \\
\hline Diabetes mellitus & 7 (21.2) & $1(12.5)$ & $2(40)$ \\
\hline \multirow[t]{6}{*}{ Co-morbidities } & $4(12.1)$ & $1(12.5)$ & $2(40)$ \\
\hline & $4(12.1)$ & $1(12.5)$ & $0(0)$ \\
\hline & $31(93.9)$ & $8(100.0)$ & $4(80)$ \\
\hline & $26(78.8)$ & $6(75.0)$ & $4(80)$ \\
\hline & $8(24.2)$ & $6(75.0)$ & $1(20)$ \\
\hline & $16(48.5)$ & $3(37.5)$ & $2(40)$ \\
\hline $\begin{array}{l}\text { Clinical } \\
\text { Presentation }\end{array}$ & $14(42.4)$ & $3(37.5)$ & $1(20)$ \\
\hline Facial pain & $1(3.0)$ & $1(12.5)$ & $0(0)$ \\
\hline Facial swelling & $3(9.1)$ & $2(25.0)$ & $1(20)$ \\
\hline Proptosis & $1(3.0)$ & $0(0)$ & $0(0)$ \\
\hline Diminution in vision & $1(3.0)$ & $0(0)$ & $1(20)$ \\
\hline
\end{tabular}

\section{DISCUSSION}

Over a one-year period, 74 patients that presented with clinical suspicion of rhinosinusitis were studied. The overall prevalence of FRS among the patients with clinical suspicion was $60.8 \%$ which was lower than a study done in the
USA in which the prevalence of FRS was $93 \%$ [11].

There was a predominance of rhinosinusitis in male patients with a male: female ratio of 1.5:1. This result was similar to the study done by Manning SC et al. [12] that also noted a male predominance with a male: female ratio of 1.6:1. 
However, a study was done by Micheal et al. [13]. showed female predominance. The results obtained in our study can be attributed to the fact that the males are more commonly exposed to irritating pollutants, have a higher prevalence of smoking and commonly go out to the fields in a hot and dry climate and may acquire frequent mucosal injuries of paranasal sinuses and acquire the agent from the field. A large number of Aspergillus conidia can lodge on the injured mucosa and can lead to an allergic form of disease whereas females have hesitance in the social setting like India to seek medical care.

In terms of age groups affected, our finding is nearer to the observation of Micheal et al. [13]. Geographically, our study comprised more of urban patients. This can be due to the fact that our institute is a tertiary care hospital. This finding is similar to the study conducted by Farhani et al. [14] where urban cases were reported more as compared to rural. Another reason could be that the population residing in urban areas are more commonly exposed to the irritant pollutants of traffic, dust, and factory residuals. These irritants cause allergic rhinitis which can progress to fungal sinusitis.

The most common presenting complaints were nasal obstruction and rhinorrhea which are comparable to the study by Irfan et al, [15]. In a similar study done in Nepal, the nasal discharge was the chief presenting symptom followed by headache and nasal blockage, either bilaterally or unilaterally [16].

Other symptoms like headache, postnasal drip, and nasal polyps were comparable to the observations of Madani et al. [17].

Diabetes mellitus and hypertension were the most common co-morbid illnesses. There are several possible reasons for these findings. A significant proportion of the Indian population lives below the poverty line and hence is malnourished. Though they are not immunocompromised in the classical sense, their poor nutritional status makes them more susceptible to these diseases. The second reason may be that diabetes mellitus, and bronchial asthma are extremely common in India and some of the patients may have had undiagnosed bronchial asthma, predisposing them to fungal sinusitis.
Prior sinus surgery also seems to be an important risk factor for the development of sinus fungal balls due to poorly ventilated sinuses [4]. In our study group, $<10 \%$ had a history of sinus surgery.

The most common type of rhinosinusitis in our study was allergic RS and the most common fungal isolate was Aspergillus flavus. This finding was similar to a 7-year audit done in a hospital in Tamil Nadu, India but contrasts with findings of Chakrabarti et al [18] who reported only a small number of patients with allergic disease in their patient groups.

Highly significant ( $p$-value $<0.05$ ) correlation was obtained between direct examination and culture. Some cases with positive direct $\mathrm{KOH}$ smear examination yielded a negative culture, which may be due to inadequate specimen or improper sample collection or antifungal therapy of the patient. Our study findings were also found comparable to a recent study conducted in 2016 in New Delhi, India [19].

Inability to perform Aspergillus-specific IgE tests for further confirmation of fungal diagnosis due to unavailability of test kits and post-treatment follow-up of the patients were some of the limitations of our study.

In conclusion, this study highlights the importance of paranasal mycosis infections in North India. The microbiological assessment is essential to confirm the diagnosis. Identification of the etiological organisms not only aids in the diagnosis and improves the management of patients, but also assists in advising the patients about the modes of spread, methods of prevention, and anticipating the possible complications. Furthermore, as certain etiological agents are more common in healthcare settings, the healthcare institutions can be directed regarding appropriate hygiene and sterility practice when relevant.

Therefore, our suggestion to clinicians is that all the patients with rhinological symptoms should be screened for microbial etiology. We believe that the present study may contribute to the effective management of rhinological infections.

\section{ACKNOWLEDGMENTS}

Financial Support: This study was performed without any financial support. 
Conflict of interest: The authors have declared no conflicts of interest.

\section{REFERENCES}

1. Kennedy DW, Thaler ER. Acute vs. chronic sinusitis: etiology, management, and outcomes. Infect Dis Clin Pract 1997; 2:49-58.

2. Das A, Bal A, Chakrabarti A, Panda N, Joshi K. Spectrum of fungal rhinosinusitis histopathologist's perspective. Histopathology 2009;54(7):854-859.

3. Taxy J. Paranasal Fungal Sinusitis: Contributions of Histopathology to Diagnosis. The Am J Surg Pathol 2006;30(6):713-720.

4. deShazo RD, O'Brien M, Chapin K, Soto-Aguilar $M$, Swain $R$, Lyons $M$, et al. Criteria for the diagnosis of sinus mycetoma. J Allergy Clin Immunol 1997;99: 475-485.

5. Morgan J, Warnock DW. Fungi. In: Browning GG, Burton MJ, Clarke R, Hibbert J, et al. ScottBrown's Otorhinolaryngology, Head and Neck Surgery. 7th ed. London: Edward Arnold; 2008; 217- 79.

6. Baker RD. Mucormycosis: A new disease? JAMA 1957;163(10):805-8.

7. Prayaga N, Srinivas M, Jadi L, Sudhakar K, Anil N. Clinical application of a microbiological study on chronic suppurative otitis media. Indian $\mathrm{J}$ Otolaryngol Head Neck Surg 2013; 2:290-4.

8. Prakash M, Lakshmi K, Anuradha S, Swathi GN. Bacteriological profile and their antibiotic susceptibility pattern of cases of CSOM. Asian J Pharm Clin Res 2013; 6:210-2.

9. Chander J. Fungal sinusitis. Textbook of Medical Mycology 3rd ed. New Delhi: Mehta Publishers; 2009 :480- 520.

10. Browning GG, Burton MJ, Clarke R, et al. Rhinosinusitis. Scott-Brown's Otorhinolaryngology, Head and Neck Surgery. $7^{\text {th }}$ ed. London: Edward Arnold; 2008. p. 1423-1467.

11. Ponikau JU, Sherris DA, Kern EB, et al. The diagnosis and incidence of allergic fungal sinusitis. Mayo Clinic Proceedings. 1999; 74:877-84.

12. Manning SC, Holman M. Further evidence for allergic pathophysiology in allergic fungal sinusitis. Laryngoscope 1998; 108:1485-96.

13. Micheal RC, Micheal JS, Ashbee RH, Mathews MS. Mycological profile of fungal sinusitis: an audit of specimens over a 7 year period in a tertiary care hospital in Tamil Nadu. Indian $\mathrm{J}$ Pathol Microbiol 2008; 51(4):493-6.

14. Farhani F, Mashouf RY, Hashemian F, Esmaeli R. Antimicrobial Resistance Patters of Aerobic Organisms in Patients with Chronic Rhinosinusitis in Hamadan, Iran. Avicenna J Clin Microb Infec 2014;1(2):e18961.

15. Irfan S, Farooq I, Fayaz W. Microbiological profile of patients with chronic sinusitis in Kashmir valley. JMS 2014;4(1):410-16.
16. Joshi RR, Bhandary S, Khanal B, Singh RK. Fungal Maxillary sinusitis: A prospective study in a tertiary care hospital of eastern Nepal. Kathmandu Univ Med J 2007;5(2):195-198.

17. Madani SA, Hashemi SA, Fazli M, Esfandiar K. Bacteriology in patients with chronic rhinosinusitis in North Iran. Jundishapur J Microbiol 2013; 6(8):e7193.

18. Chakrabarti A, Sharma SC, Chander J. Epidemiology and pathogenesis of paranasal sinus mycoses. Otolaryngol Head Neck Surg 1992;107:745-750.

19. Kaur R, Lavanya S, Khurana N, Gulati A, Dhakad MS. Allergic Fungal Rhinosinusitis: A Study in a Tertiary Care Hospital in India. J Allergy (Cairo) 2016; 2016:7698173. 